\title{
Revised and Extended Analysis of Five Times lonized Argon (Ar VI)
}

\author{
M. Raineri, F. Bredice, M. Gallardo and J. G. Reyna Almandos \\ Centro de Investigaciones Opticas, (CIOp), Casilla de Correo 124, 1900 La Plata, Argentina
}

\section{J. B. Pagan}

Instituto de Física "Gleb Wataghin", Universidade Estadual de Campinas (UNICAMP), C.P. 6165, 13081 Campinas, São Paulo, Brasil

and

\author{
A. G. Trigueiros* \\ Department of Physics, University of Lund, S-223 62 Lund, Sweden
}

Received September 20, 1991; accepted November 22, 1991

\begin{abstract}
The spectrum of five times ionized argon, (Ar VI), has been observed in the $280-2100 \AA$ wavelength range. Eighty-seven lines have been identified as transitions between levels of the $3 s^{2} 3 p, 3 s 3 p^{2}, 3 s^{2} 3 d, 3 p^{3}, 3 s 3 p 3 d, 3 s^{2} 4 s$, $3 s^{2} 4 d$ and $3 s 3 p 4 s$ configurations. For 33 of the lines the classification is new. Forty-one energy level values belonging to these configurations were analyzed and we propose 9 new energy level values for levels corresponding to odd parity configurations. The configurations are interpreted by fitting the theoretical energy expressions to the observed energy levels using leastsquares techniques. The parameter values are compared with results from Hartree-Fock calculations.
\end{abstract}

\section{Introduction}

The ground-state configuration of five times ionized argon, $\left(\mathrm{Ar}^{5+}\right.$ ), is $3 s^{2} 3 p$ with the term ${ }^{2} \mathrm{P}$. Ar VI belongs to the Al I isoelectronic sequence. Excited states either belong to simple one-electron configuration of the type $3 s^{2} n l$ or to threeelectron configurations such as $3 s 3 p^{2}, 3 p^{3}$ and $3 s 3 p\left({ }^{1,3} P\right) n l$ etc., giving both doublets and quartets.

The spectra of the first, second and third elements in this sequence are presented in Atomic Energy Levels (AEL), Ref. [1]. Subsequent to this tabulation, the Al I spectrum was investigated by Eriksson and Isberg [2]. Results about Si II were published by Shenstone [3] and the P III spectrum was studied by Magnusson and Zetterberg [4]. Early results on the spectra of $\mathrm{S} I V$ and $\mathrm{Cl} \mathrm{V}$ have been published in AEL but later results of S IV were compiled by Martin et al., see Ref. [5] and references therein. The first results about the spectrum of Ar VI in the vacuum ultraviolet were published by Phillips and Parker [6]. Fawcett et al. [7] studied the spectra of multiple ionized inert gases, including Ar, and Schönheit [8] made a similar study finding a large quantity of new lines. Lines corresponding to the spinforbidden resonance multiplet $3 s^{2} 3 p^{2} P-3 s 3 p^{24} P$ were reported by Ekberg and Svensson [9]. Using a Theta-Pinch light source Fawcet $e t$ al. [10] classified some lines of Ar VI.

\footnotetext{
- Permanent address: Instituto de Fisica "Gleb Wataghin", Universidade Estadual de Campinas (UNICAMP), C.P. 6165, 13081 Campinas, São Paulo, Brasil.
}

Using the beam-foil technique Livingston et al. [11] have studied the argon spectra from Ar V to Ar VIII and using the same technique Buchet-Poulizac et al. [12] have studied the argon spectra from Ar VI to Ar VIII. A few lines of Ar VI were classified in the work of De-Ye et al. [13].

Theoretical calculations for the Al I isoelectronic sequence were made by Fawcett [14]. Computed ab-initio transition probabilities and energy levels for $\mathrm{Al} \mathrm{I-like} \mathrm{ions}$ were made by Huang [15] and data about highly ionized copper and zinc, belonging to this sequence, were published by Sugar and Kaufman [16-17].

Recently, Träbert et al. [18-19] have studied the spectrum of argon and other ions in the vacuum ultraviolet. They searched for lines of $\mathrm{Mg}$-, $\mathrm{Al}$ - and Si-like ions. Recoil ion spectroscopy was used by Lesteven-Vaïsse et al. [20] who have studied all argon spectra from Ar I to Ar IX. Some anomalies in resonance transitions in the Al I isoelectronic sequence were observed by Engström et al. [21] and a new work about energy levels and lifetimes of AT VI was recently published by Pinnington et al. [22]. An extended analysis of spectra and term systems in Al-like Ca VIII to Ni XVI was published by Redfors and Litzén [23] and lifetimes of the $3 s^{2} 4 s^{2} S$ states for Al-like ions from S IV to Fe XIV were published by Thornbury et al. [24]. Transitions in spectra of highly ionized $\mathrm{Kr}$ and Mo belonging to the $\mathrm{Al} \mathrm{I}$ isoelectronic sequence were recently reported by Jupén et al. [25]. In the present work we report a revised and extended analysis of Ar VI that includes 33 newly classified lines and 9 new energy level values.

\section{Experiment}

The light source used in the present work is a discharge tube built at the Centro de Invesitgaciones Opticas, (CIOp), to study highly ionized gases [26]. It is a $30 \mathrm{~cm}$ long Pyrex tube with an inner diameter of $3 \mathrm{~mm}$. Gas excitation was produced by discharging a bank of low inductance capacitors varying between 2.5 and $100 \mathrm{nF}$ and charged up to $19 \mathrm{kV}$ through the tube. Light radiation emitted axially was analyzed using a $3 \mathrm{~m}$ normal incidence vacuum spectro- 
graph with a concave diffraction grating of 1200 lines $/ \mathrm{mm}$, blazed for $1200 \AA$. The plate factor in the first order is $2.77 \AA / \mathrm{mm}$. Ilford Q-2 plates were used to record the spectra between 280-2100 A. C III and N II [27], O III [28] and lines of Ar III-Ar V [27] were recorded as internal wavelength standards. Exposing the plates with $10^{4}$ shots we were able to obtain good spectra of argon below $300 \AA$. The lines were observed in the first, second, and in some cases, third order of diffraction.

The gas pressure, the discharge voltage, and the capacitance were varied to distinguish between different stages of ionization. A well developed Ar VI spectrum was obtained with the following parameters: $120 \mathrm{mTorr}, 18 \mathrm{kV}$ and $20 \mathrm{nF}$. The positions of spectral lines on the plates were determined with a rotating prism photoelectric automatic Grant comparator whose precision is $1 \mu \mathrm{m}$. The uncertainty in determining the wavelength of unperturbed lines by this procedure is estimated to be $\pm 0.01 \AA$ in the first diffraction order.

\section{Analysis}

The Ar VI lines observed in the present work are given in Table I, 33 of them being without previous classification. The intensities of the lines given in the table are based on visual estimates and the values given in the calculated column are deduced from the optimized level values. The optimized energy values derived from the observed lines are given in Table II and the general structure of the term system is shown in Fig. 1. The level values were determined in an iterative procedure where the wavenumbers of the observed lines are weighted according to their estimated uncertainties. Theoretical predictions of the structure of the

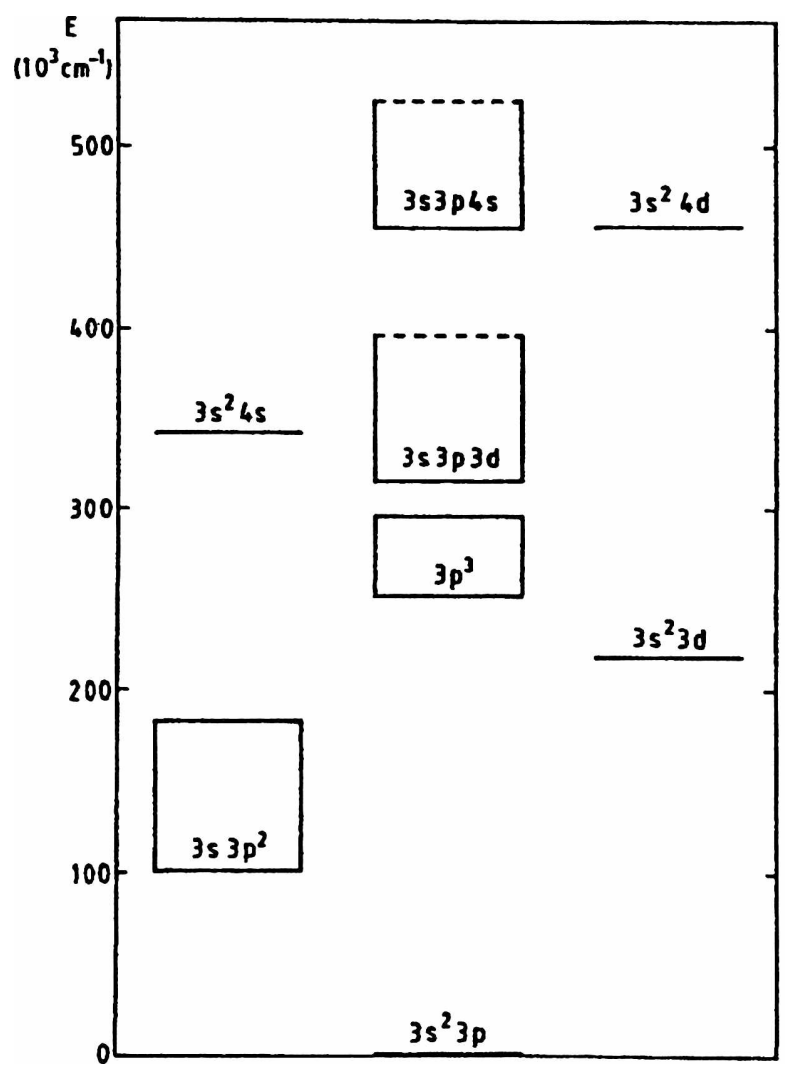

Fig. I. Gross structure of the observed Ar VI configurations

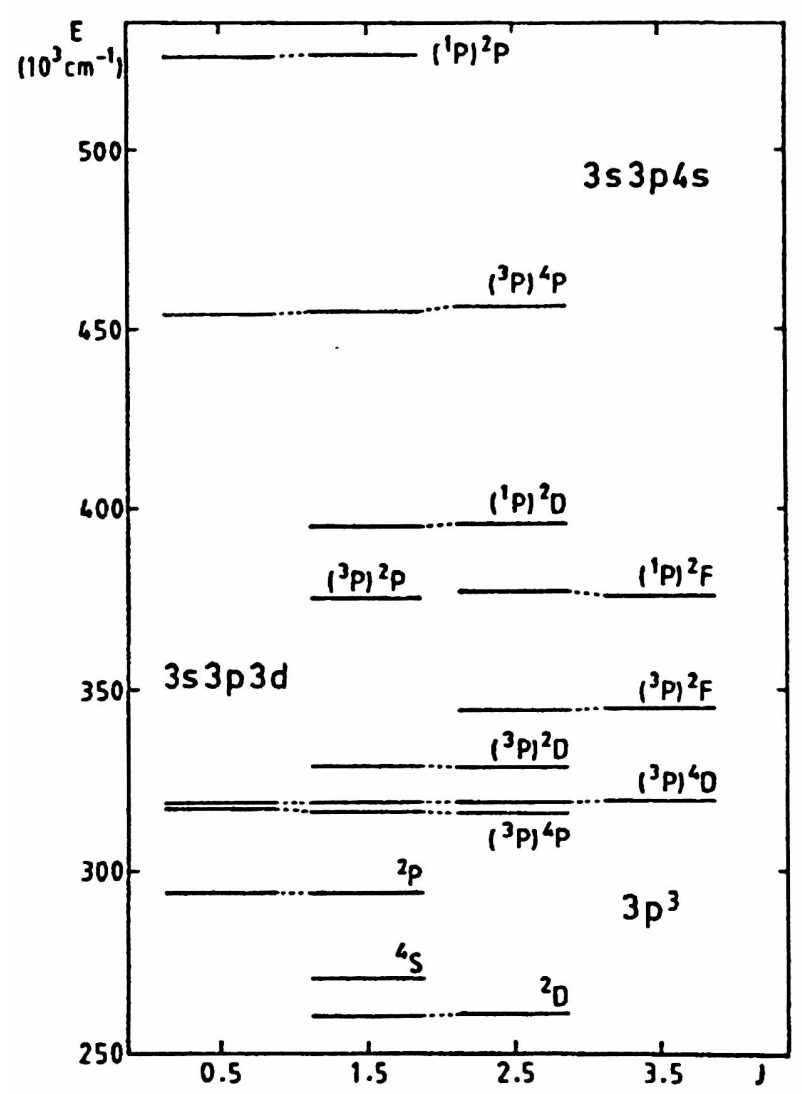

Fig. 2. Level structure of the observed $3 p^{3}, 3 s 3 p 3 d$ and $3 s 3 p 4 s$ configurations in Ar VI

configurations made at the Department of Physics, University of Lund, were also used in the analysis. The predictions were obtained by diagonalizing the energy matrices with appropriately scaled Hartree-Fock (HF) values for the energy parameters. The computer code developed by Cowan [29] was used. All levels designations in Table II are in $L S$ notation, and in the same table we present the percentage composition of the levels.

A comparison with the level system given by Pinnington et al. [22] shows that six of their level values have been confirmed, although the accuracy has been considerably improved. However, for seven levels we propose new identifications as discussed below.

The observed structure of the configurations $3 p^{3}, 3 s 3 p 3 d$ and $3 s 3 p 4 s$ is shown in Fig. 2 . For the level $3 s 3 p\left({ }^{3} P\right) 3 d^{2} F_{5 / 2}$ we propose the new value $344307.9 \mathrm{~cm}^{-1}$. The level is established by five new lines that are given in Table 1 .

For the level $3 s 3 p\left({ }^{3} P\right) 3 d^{2} F_{7 / 2}$ we propose the new value $346073.4 \mathrm{~cm}^{-1}$ determined by two new lines, see Table I. Both level values fit well with the extrapolation that can be done from the isoelectronic data published in Ref. [23].

For the level $3 s 3 p\left({ }^{3} P\right) 3 d^{2} P_{3 / 2}$ we propose the new value $375657.6 \mathrm{~cm}^{-1}$. This level is determined by four new lines, see Table I. The level value fits very well with the isoelectronic trend from Ref. [23]. The isoelectronic graph gives for the $3 s 3 p\left({ }^{3} P\right) 3 d^{2} P_{1 / 2}$ level a probable value near $376300 \mathrm{~cm}^{-1}$. We were not able to find the transitions establishing this level, but according to the extrapolated values mentioned above and our theoretical predictions we reject the experimental value published in the work of Pinnington et al. [22] for this level. 
Table I. Classified lines in the Ar VI spectrum

\begin{tabular}{|c|c|c|c|c|c|c|c|c|c|c|c|}
\hline \multirow[b]{2}{*}{ Int. } & \multirow[b]{2}{*}{$\lambda(A)$} & \multicolumn{2}{|l|}{$\sigma\left(\mathrm{cm}^{-1}\right)$} & \multirow{2}{*}{\multicolumn{2}{|c|}{ Transition }} & \multirow[b]{2}{*}{ Int. } & \multirow[b]{2}{*}{$\lambda(\boldsymbol{A})$} & \multicolumn{2}{|l|}{$\sigma\left(\mathrm{cm}^{-1}\right)$} & \multirow{2}{*}{\multicolumn{2}{|c|}{ Transition }} \\
\hline & & Observed & Calculated & & & & & Observed & Calculated & & \\
\hline 4 & $219.91^{\text {ct }}$ & 454736.6 & 45.6 & $3 s^{2} 3 p^{2} P_{1 / 2}$ & $-3 s^{2} 4 d^{2} D_{3 / 2}$ & 4 & $520.61^{2.5}$ & 1920827 & 1.5 & $3 s 3 p^{2} P_{3 / 2}$ & $\left.-3 s 3 \not x^{3} P\right) 3 d^{2} P_{3 / 2}$ \\
\hline 6b & $220.93^{c 4}$ & 4526227 & 591.7 & $3 s^{2} 3 p^{2} P_{3 / 2}$ & $-3 s^{2} 4 d^{2} D_{s / 2}$ & 10 & $544.73 \circ 4$ & 183576.3 & 6.1 & $3 s^{2} 3 p^{2} P_{1 / 2}$ & $-3 s 3 p^{2}{ }^{2} P_{3 / 2}$ \\
\hline 1 & $281.43^{c .4}$ & 355321.2 & 16.8 & $3 s 3 p^{2+} P_{3 / 2}$ & $-3 s 3 p\left(^{3} P\right) 4 s^{4} P_{9 / 2}$ & 10 & $548.91^{\circ .4}$ & 182179.2 & 81.1 & $3 s^{2} 3 p^{2} P_{t / 2}$ & $-353 p^{2}{ }^{2} P_{1 / 2}$ \\
\hline 1 & $281.91^{<4}$ & 354723.8 & 14.3 & $353 p^{24} P_{1 / 2}$ & $\left.-3 s 3 p P^{3} P\right) 4 s^{4} P_{3 / 2}$ & $6 \mathrm{bd}$ & $55135^{4}$ & 181373.6 & 69.0 & $3 s^{2} 3 p^{2} P_{3 / 2}$ & $-3 s 3 p^{21} P_{3 / 2}$ \\
\hline 2 & $282.42^{5.4}$ & 354077.9 & 822 & $3 s 3 p^{2+4} P_{S / 2}$ & $\left.-3 s 3 p p^{3} P\right) A s^{4} P_{g / 3}$ & 8 & $555.63^{\circ .4}$ & 179975.5 & 4.0 & $3 s^{2} 3 p^{2} P_{3 / 2}$ & $-3 s 3 p^{22} P_{1 / 2}$ \\
\hline 1 & $282.55^{\mathrm{c} .4}$ & 353917.7 & 7.7 & $3 s 3 p^{2+} P_{3 / 2}$ & $\left.-3 s 3 p r^{3} P\right) 4 s^{4} P_{3 / 2}$ & 4 & $564.30^{\circ}$ & 177209.9 & 11.1 & $3 s^{2} 3 d^{2} D_{3 / 2}$ & $\left.-3 s 3 P x^{2} P\right) 3 d^{2} D_{s / 2}$ \\
\hline 1 & $283.16^{6.4}$ & 353155.0 & 5.0 & $3 s 3 p^{2+} P_{3 / 2}$ & $-3 s 3 p\left(^{3} P\right) 4 s^{4} P_{1 / 2}$ & 6 & $564.49^{1, x}$ & 177151.9 & 0.3 & $3 s^{2} 3 d^{2} D_{3 / 2}$ & $\left.-353 d^{1} P\right) 3 d^{2} D_{s / 2}$ \\
\hline 1 & $283.55^{\circ}$ & 352676.0 & 83.1 & $3 s 3 p^{2} P_{s / 2}$ & $\left.-\left.3 s 3 p\right|^{3} P\right) 4 s^{4} P_{3 / 2}$ & 6ы & $565.29^{\circ}$ & 176901.4 & 899.0 & $3 s^{2} 3 d^{2} D_{3 / 2}$ & $\left.-3 s 3 P X^{1} P\right) 3 d^{2} D_{s / 2}$ \\
\hline 2 & $294.03^{2.4}$ & 340095.7 & 5.7 & $3 s^{2} 3 p^{2} P_{3 / 2}$ & $-3 s^{2} 4 s^{2} s_{1 / z}$ & 4 & $565.49 \mathrm{es}$ & 176838.4 & 92 & $3 s^{2} 3 d^{2} D_{s / 2}$ & $\left.-3 s 3 p x^{1} P\right) 3 d^{2} D_{3 / 2}$ \\
\hline $2 \mathbf{b l}$ & 326.150 .8 & 306609.2 & 598.3 & $3 s^{2} 3 d^{2} D_{3 / 2}$ & $\left.-3 s 3 P r^{1} P\right) 4 s^{2} P_{3 / 2}$ & 8 & $587.02^{24}$ & 170351.5 & 22 & $3 s 3 p^{24} P_{1 / 2}$ & $-3 p^{34} s_{312}$ \\
\hline 4 & $326.22^{\circ .4}$ & 306537.4 & 8.5 & $3 s^{2} 3 d^{2} D_{s / 2}$ & $-3 s 3 p\left({ }^{1} P / 4 s^{2} P_{3 / 2}\right.$ & $8 w$ & 588.91 cd & 169803.9 & 3.7 & $3 s^{2} 3 p^{2} P_{1 / 2}$ & $-3 s 3 p^{21} S_{1 / 2}$ \\
\hline 4 & $326.32^{\circ .4}$ & 306448.7 & 50.6 & $3 s^{2} 3 d^{2} D_{3 / 2}$ & $-3 s 3 p\left(^{1} P\right) 4 s^{2} p_{u z}$ & 8 & $589.78^{\circ .4}$ & 169555.4 & 5.6 & $3 s 3 p^{2+4} P_{3 / 2}$ & $-3 p^{3} s_{3 / 2}$ \\
\hline 6 & $409.10^{\circ}$ & 244440.4 & 0.1 & $3 s 3 p^{22} D_{3 / 2}$ & $\left.-3 s 3 p x^{1} P\right] 3 d^{2} F_{s / 2}$ & 8 & $594.10^{c .4}$ & 168322.2 & 1.0 & $3 s 3 p^{2}+P_{3 / 2}$ & $-3 p^{3} s_{3 / 2}$ \\
\hline 2 & $409.28^{\circ .6}$ & 244328.9 & 7.4 & $3 s 3 p^{22} D_{s / 2}$ & $\left.-3 s^{3} \beta \alpha^{\prime} P\right) 3 d^{2} F_{s / 2}$ & 6w & $-596.67^{4}$ & 167596.8 & 6.6 & $3 s^{2} 3 p^{2} P_{3 / 2}$ & $-3 s 3 p^{22} s_{1 / 2}$ \\
\hline $6 \mathrm{bl}$ & $410.10^{\circ .5}$ & 2438424 & 3.1 & $3 s 3 p^{2}{ }^{2} D_{3 / 2}$ & $-3 s 3 x x^{1} P \mid 3 d^{2} F$, & $8 \mathrm{bl}$ & $618.67^{6.5}$ & 161637.5 & 8.1 & $3 s 3 p^{21} D_{3 / 2}$ & $-3 p^{12} P_{1 / 2}$ \\
\hline $6 \mathrm{~A}$ & $455.83^{6.4}$ & 219379.6 & 8.3 & $3 s 3 p^{24} P_{1 / 2}$ & $\left.-3 s 3 p d^{3} P\right) 3 d^{4} D_{3 / 2}$ & $6 \mathrm{bl}$ & $618.72^{\text {a.e }}$ & 161624.0 & 3.4 & $3 s 3 p^{2} D_{1 / 2}$ & $-3 p^{32} P_{3 / 2}$ \\
\hline 6 & $456.38^{\mathrm{cs}}$ & 219113.5 & 3.8 & $3 s 3 p^{24} P_{1 / 2}$ & $\left.-3 s 3 p p^{3} P\right) 3 d^{4} D_{1 / 2}$ & 8 & $619.16^{6.5}$ & 161508.7 & 10.7 & $3 s 3 p^{2} D_{s / 2}$ & $-3 p^{3}{ }^{2} P_{3 / 2}$ \\
\hline 8 & $457.01^{. .4}$ & 218812.2 & 2.2 & $3 s 3 p^{24} P_{3 / 2}$ & $-3 s 3 p^{3} P / 3 d^{4} D_{3 / 2}$ & 6 & $622.15^{2 x}$ & 160732.1 & 1.8 & $3 s 3 p^{22} P_{3 / 2}$ & $\left.-3 s 3 p x^{3} P\right) 3 d^{2} F_{s / 2}$ \\
\hline $8 \mathbf{b l}$ & $457.46^{c .4}$ & 218.598 .3 & 3.0 & $3 s^{2} 3 p^{2} P_{1 / 2}$ & $-3 s^{2} 3 d^{2} D_{3 / 2}$ & 6 & $631.68^{\circ}$ & 158307.9 & 8.5 & $3 s^{2} 3 d^{2} D_{3 / 2}$ & $-3 s 3 p p^{1} P 73 d^{2} F_{s / 2}$ \\
\hline $4 \mathrm{bl}$ & $457.51^{0.6}$ & 218574.5 & 81.7 & $3 s 3 p^{24} P_{3 / 2}$ & $-3 s 3 p d^{3} P \mid 3 d^{4} D_{3 / 2}$ & 4 & $631.91^{1 . c}$ & 158248.9 & 8.7 & $3 s^{2} 3 d^{2} D_{s / 2}$ & $\left.-3 s 3 \alpha^{1} P\right) 3 d^{2} D_{3 / 2}$ \\
\hline $8 \mathrm{~b}$ & $458.05<4$ & 218318.6 & 72 & $3 s 3 p^{24} P_{3 / 2}$ & $\left.-3 s 3 p d^{3} P\right) 3 d^{4} D_{1 / 2}$ & $6 \mathrm{~A}$ & $633.85^{\circ}$ & 157765.0 & 4.4 & $3 s^{2} 3 d^{2} D_{s / 2}$ & $\left.-3 s 3 \mathcal{X}^{1} P\right) 3 d^{2} F_{7 / 2}$ \\
\hline $8 \mathbf{w}$ & $459.32^{\star 4.4}$ & 217714.5 & 4.5 & $3 s 3 p^{2}{ }^{4} p_{9 / 2}$ & $-3 s 3 p\left({ }^{3} P\right) 3 d^{4} D y_{12}$ & 4 & $63694^{4.6}$ & 157001.5 & 4.8 & $3 s^{2} 3 d^{2} D_{s / 2}$ & $\left.-3 s 3 p x^{3} P\right) 3 d^{2} P_{3 / 2}$ \\
\hline 6 & $460.09^{6.4}$ & 217346.0 & 7.1 & $3 s 3 p^{24} P_{s / 2}$ & $\left.-3 s 3 p p^{3} P\right) 3 d^{4} D_{3 / 2}$ & 6 & $687.84^{\prime}$ & 145382.8 & 2.7 & $3 s 3 p^{21} P_{3 / 2}$ & $\left.-3 s 3 R^{3} P\right) 3 d^{2} D_{s / 2}$ \\
\hline 6 & $460.19 . .4$ & 217300.7 & 0.6 & $3 s 3 p^{2}+P_{1 / 2}$ & $\left.-3 s^{3} p p^{3} P\right) 3 d^{4} P_{1 / 2}$ & $10 w$ & $754.93^{\mathrm{e} . \mathrm{s}}$ & 132462.1 & 1.4 & $3 s^{2} 3 p^{2} P_{1 / 2}$ & $-3 s 3 p^{22} D_{3 / 2}$ \\
\hline $8 w$ & $461.23^{2 . d}$ & 216811.3 & 2.3 & $3 \times 3 p^{2}+P_{1 / 2}$ & $\left.-3 s 3 p^{3} P\right) 3 d^{4} P_{3,2}$ & 6A & $767.07^{8,4}$ & 1303663 & 7.0 & $3 s^{2} 3 p^{2} P_{3 / 2}$ & $-3 s 3 p^{22} D_{s / 2}$ \\
\hline 4 & $461.89^{6}$ & 216501.8 & 504.0 & $3 s 3 p^{24} P_{3 / 2}$ & $\left.-3 s 3 p^{3} P\right) 3 d^{4} P_{1 / 2}$ & 10 & $767.73^{\text {c.d }}$ & 130253.2 & 4.3 & $3 s^{2} 3 p^{2} P_{3 / 2}$ & $-3 s 3 p^{22} D_{3 / 2}$ \\
\hline 6 & $462.01^{\circ}$ & 216445.3 & 5.7 & $3 s^{2} 3 p^{2} P_{3 / 2}$ & $-3 s^{2} 3 d^{2} D_{3 / 2}$ & 6 & $78241^{I}$ & 127810.7 & 0.0 & $3 s 3 p^{22} D_{3 / 2}$ & $-3 p^{32} D_{s / 2}$ \\
\hline 6 & $462.13^{4}$ & 216386.7 & 5.9 & $3 s^{2} 3 p^{2} P_{3 / 2}$ & $-3 s^{2} 3 d^{2} D_{3 / 2}$ & $\mathbf{8 A}$ & $783.07^{\prime}$ & 1277025 & 697.3 & $3 s 3 p^{22} D_{s i 2}$ & $-3 p^{3} D_{s / 2}$ \\
\hline 2 & $462.93^{\circ}$ & 216013.8 & 5.7 & $3 s 3 p^{2}{ }^{4} P_{3 / 2}$ & $\left.-3 s 3 P^{3} P\right) 3 d^{4} P_{3 / 2}$ & $\mathbf{8 A}$ & $783.66^{\prime}$ & 127606.2 & 5.5 & $3 s 3 p^{21} D_{3 / 2}$ & $-3 p^{3} D_{3 / 2}$ \\
\hline 8 & $464.26^{6, .}$ & 215397.0 & 6.8 & $3 s 3 p^{24} P_{3 / 2}$ & $\left.-3 s 3 p^{3} P\right] 3 d^{4} P_{s / 2}$ & 6 & $784.35^{t}$ & 127494.0 & 2.8 & $3 s 3 p^{22} D_{s / 2}$ & $-3 p^{32} D_{3 / 2}$ \\
\hline 6 & 465.58 -. & 214783.2 & 1.1 & $3 s 3 p^{2}<P_{s / 2}$ & $\left.-3 s^{3} p^{3} P\right) 3 d^{4} P_{3 / 2}$ & 6 & $784.80^{\circ}$ & 127420.9 & 0.6 & $3 s^{2} 3 d^{2} D_{s / 2}$ & $\left.-3 s 3 p d^{3} P\right) 3 d^{2} F_{t / 2}$ \\
\hline 8 & $466.94^{\text {ced }}$ & 214161.5 & 22 & $3 s 3 p^{2}<P_{s / 2}$ & $\left.-3 s 3 p d^{3} P\right) 3 d^{4} P_{s / 2}$ & $6 \mathrm{~A}$ & $795.44^{\circ}$ & 125716.8 & 4.9 & $35^{2} 3 d^{2} D_{3 / 2}$ & $\left.-3 s 3 R^{3} P\right) 3 d^{2} F_{s / 2}$ \\
\hline 4 & $468.39^{\circ}$ & 213498.6 & 9.3 & $3 s 3 p^{2} D_{s / 2}$ & $\left.-3 s 3 p^{3} P\right) 3 d^{2} F_{7 / 2}$ & $6 \mathrm{~d}$ & $795.85^{\circ}$ & 125651.8 & 5.1 & $3 s^{2} 3 d^{2} D_{3 / 2}$ & $\left.2-3 s^{3} 3 p^{3} P\right] 3 d^{2} F_{s / 2}$ \\
\hline 6 & $468.80^{\circ, .6}$ & 213310.3 & 0.9 & $3 s 3 p^{22} P_{1 / 2}$ & $\left.-3 s 3 d^{2} P\right) 3 d^{2} D_{3 / 2}$ & 8 & $804.62^{\prime}$ & 124282.3 & 1.1 & $3 s 3 p^{22} S_{1 / 2}$ & $-3 p^{32} p_{3 / 2}$ \\
\hline 8 & 471.19.x & 212227.8 & 7.9 & $3 s 3 p^{22} P_{3 / 2}$ & $\left.-3 s 3 p d^{1} P\right) 3 d^{2} D_{3 / 2}$ & 8 & $893.50^{\circ}$ & 111919.3 & 8.4 & $3 s 3 p^{22} P_{1 / 2}$ & $-3 p^{32} P_{1 / 2}$ \\
\hline $4 A$ & $471.87^{\star}$ & 211922.6 & 15.9 & $3 s 3 p^{22} P_{3 / 2}$ & $-3 s 3 p\left(^{1} P\right) 3 d^{2} D_{3 / 2}$ & $6 \mathrm{bl}$ & $893.63^{\prime}$ & 111903.3 & 3.7 & $353 p^{22} P_{1 / 2}$ & $-3 p^{32} p_{3 / 2}$ \\
\hline 6 & $472.04^{-2}$ & 211844.4 & 6.5 & $3 s 3 p^{2} D_{3 / 2}$ & $\left.-3 s 3 p d^{3} P\right) 3 d^{2} F_{s / 2}$ & 6 & $904.90^{\mathrm{t}}$ & 110509.0 & 8.7 & $3 s 3 p^{22} P_{3 / 2}$ & $-3 p^{32} P_{3 / 2}$ \\
\hline 4 & 472.29 .e & 211735.2 & 3.8 & $3 s 3 p^{22} D_{s / 2}$ & $-3 s 3 p\left({ }^{3} P\right) 3 d^{2} F_{s / 2}$ & 8 & $998.43^{d}$ & 100157.6 & 8.2 & $3 s 3 p^{22} P_{2 / 2}$ & $-3 p^{3}{ }^{4} p_{1 / 2}$ \\
\hline 6 & $485.78^{-.5}$ & 205853.5 & 3.9 & $3 s 3 p^{22} S_{1 / 2}$ & $-3 s 3 p\left(x^{3} P\right) 3 d^{2} P_{3 / 2}$ & 1 & $1000.16^{2.4}$ & 99983.8 & 2.3 & $3 s 3 p^{22} P_{3 / 2}$ & $-3 p^{3}{ }^{4} p_{5 / 2}$ \\
\hline 8 & $508.83^{c, *}$ & 196528.6 & 8.5 & $3 s 3 p^{22} D_{3 / 2}$ & $-3 s 3 p\left(^{3} P\right) 3 d^{2} D_{3 / 2}$ & 6 & $1012.66^{4}$ & 98749.4 & 7.7 & $3 s 3 p^{22} P_{3 / 2}$ & $-3 p^{3}{ }^{4} P_{3 / 2}$ \\
\hline 8 & $508.91^{\text {the }}$ & 196496.9 & 7.4 & $3 s 3 p^{22} D_{3 / 2}$ & $-3 s 3 p\left({ }^{3} P\right) 3 d^{2} D_{s / 2}$ & $10 w$ & 1303.87 & 76694.6 & 5.3 & $3 s 3 p^{22} P_{3 / 2}$ & $-3 p^{32} D_{3 / 2}$ \\
\hline $4 \mathrm{bl}$ & $509.12^{\star}$ & 196417.0 & 5.8 & $3 s 3 p^{22} D_{s / 2}$ & $\left.-3 s 3 P f^{3} P\right) 3 d^{2} D_{3 / 2}$ & 4 & $1420.60^{\circ}$ & 703925 & 2.5 & $3 s^{2} 4 d^{2} D_{s / 2}$ & $\left.-3 s^{3} P R^{1} P\right) 4 s^{2} P_{3 r}$ \\
\hline 8 & $509.20^{<-5}$ & 196385.3 & 4.7 & $3 s 3 p^{22} D_{3 / 2}$ & $-3 s 3 p\left(^{3} P\right) 3 d^{2} D_{s / 2}$ & 4 & $1422.51^{\circ}$ & 70298.0 & 8.0 & $3 s^{2} 4 d^{2} D_{3 / 2}$ & $-3 s 3 p\left({ }^{1} P\right) 4 s^{2} P_{1 / 2}$ \\
\hline 4 & $516.86^{\circ .5}$ & 193476.2 & 6.5 & $3 s 3 p^{22} P_{1 / 2}$ & $\left.-3 s 3 P^{3} P\right) 3 d^{2} P_{3 / 2}$ & & & & & & \\
\hline
\end{tabular}

- New line

- Previously identified by Lesteven-Vaïsse (Ref. [20])

- Measured in second order

- Previously identified according to Ref. [27]

- Previously identified by De-Ye et al. (Ref. [13])

'Previously identified by Pinnington et al. (Ref. [22])

$A=$ Asimetric line

$\mathbf{w}=$ Wide line

bl = Blended line

For the levels $3 s 3 p\left({ }^{1} P\right) 3 d^{2} D_{3 / 2}$ and $3 s 3 p\left({ }^{1} P\right) 3 d^{2} D_{s / 2}$ we propose the new values $395492.0 \mathrm{~cm}^{-1}$ and $395804.1 \mathrm{~cm}^{-1}$ respectively. These level values are in accordance with the values obtained along the isoelectronic sequence using data of Ref. [23] and with our theoretical predictions. The $3 s 3 p\left({ }^{1} P\right) 3 d^{2} D_{3 / 2}$ level is determined by four new lines, see Table I. For the other level of the same multiplet we classified three lines that are given in the same table.

For the $3 s 3 p\left({ }^{1} P\right) 3 d^{2} F_{7 / 2}$ level we propose the new value $376417.2 \mathrm{~cm}^{-1}$. This level is determined by a new line at $410.10 \AA$, classified as $3 s 3 p^{22} D_{5 / 2}-3 s 3 p\left({ }^{1} P\right) 3 d^{2} F_{7 / 2}$ and another new line at $633.85 \AA$, classified as $3 s^{2} 3 d^{2} D_{s / 2}-3 s 3 p\left({ }^{1} P\right) 3 d^{2} F_{7 / 2}$. For the $3 s 3 p\left({ }^{1} P\right) 3 d^{2} F_{5 / 2}$ level we propose the new value $376901.5 \mathrm{~cm}^{-1}$. The level is determined by four new lines that are classified in Table I. Both level values of this multiplet are in good agreement with our theoretical predictions and with the graphic extrapolation along the isoelectronic sequence based on the work of Ref. [23].

For the level $3 s 3 p\left({ }^{1} P\right) 4 s^{2} P_{1 / 2}$ we propose the new value $525043.6 \mathrm{~cm}^{-1}$. The level is determined by a new line at $326.32 \AA$, classified as $3 s^{2} 3 d^{2} D_{3 / 2}-3 s 3 p\left({ }^{1} P\right) 4 s^{2} P_{1 / 2}$, and 
Table II. Energy levels of Ar VI

\begin{tabular}{|c|c|c|c|c|c|}
\hline \multirow{2}{*}{$\frac{\text { Designation }}{3 s^{2} 3 p^{2} P_{1 / 2}}$} & \multicolumn{2}{|c|}{ Energy $\left(\mathrm{cm}^{-1}\right)$} & & \multicolumn{2}{|c|}{ Percentage composition" } \\
\hline & 0.0 & 97 & & & \\
\hline $3 s^{2} 3 p^{2} P_{3 / 2}^{1 / 2}$ & 2207.1 & 97 & & & \\
\hline $3 s 3 p^{2} 4 P_{1 / 2}$ & 100158.2 & 99 & & & \\
\hline $3 s 3 p^{24} P_{3 / 2}^{1 / 2}$ & 100954.8 & 99 & & & \\
\hline $3 s 3 p^{24} P_{s / 2}$ & 102189.2 & 99 & & & \\
\hline $3 s 3 p^{22} D_{3 / 2}$ & 132461.4 & 87 & & $3 s 3 p^{22} D$ & $+123 s^{2} 3 d^{2} D$ \\
\hline $3 s 3 p^{2}{ }^{2} D_{s / 2}$ & 132574.1 & 87 & & $3 s 3 p^{22} D$ & $+123 s^{2} 3 d^{2} D$ \\
\hline $3 s 3 p^{22} S_{1 / 2}$ & 169803.7 & 99 & & & \\
\hline $3 s 3 p^{22} P_{1 / 2}$ & 182181.1 & 99 & & & \\
\hline $3 s 3 p^{22} P_{3 / 2}$ & 183576.1 & 99 & & & . \\
\hline $3 s^{2} 3 d^{2} D_{3 / 2}$ & 218593.0 & 87 & & $3 s^{2} 3 d^{2} D$ & $+123 s 3 p^{22} D$ \\
\hline $3 s^{2} 3 d^{2} D_{s / 2}$ & 218652.8 & 87 & & $3 s^{2} 3 d^{2} D$ & $+123 s 3 p^{22} D$ \\
\hline $3 p^{32} D_{3 / 2}$ & 260066.9 & 64 & & $3 p^{32} D$ & $+353 s 3 p\left({ }^{3} P\right) 3 d^{2} D$ \\
\hline $3 p^{32} D_{3 / 2}$ & 260271.4 & 64 & & $3 p^{32} D$ & $+353 s 3 p\left({ }^{3} P\right) 3 d^{2} D$ \\
\hline $3 p^{34} S_{3 / 2}$ & 270510.4 & 99 & & & \\
\hline $3 p^{32} P_{3 / 2}$ & 294084.8 & 73 & & $3 p^{32} P$ & $+193 s 3 p\left({ }^{3} P\right) 3 d^{2} P$ \\
\hline $3 p^{32} P_{1 / 2}$ & 294099.5 & 74 & . & $3 p^{32} P$ & $\begin{array}{l}+63 s 3 p\left({ }^{3} P\right) 3 d^{2} P \\
+193 s 3 p\left(^{3} P\right) 3 d^{2} P \\
+63 s 3 p\left({ }^{2} P\right) 3 d^{2} P\end{array}$ \\
\hline $3 s 3 p\left({ }^{3} P\right) 3 d^{4} P_{5 / 2}$ & 316351.6 & 98 & & & \\
\hline $\left.3 s^{3} p^{3} P\right) 3 d^{4} P_{3 / 2}$ & 316970.5 & 97 & & & \\
\hline $3 s 3 p\left({ }^{3} P\right) 3 d^{4} P_{1 / 2}$ & 317458.8 & 98 & & & \\
\hline $\left.3 s 3 p p^{3} P\right) 3 d^{4} D_{1 / 2}$ & 3192720 & 98 & & & \\
\hline $3 s 3 p\left({ }^{3} P\right) 3 d^{4} D_{3 / 2}$ & 319536.5 & 97 & & & \\
\hline $3 s 3 p\left({ }^{3} P\right) 3 d^{4} D_{s / 2}$ & 319767.0 & 98 & & & \\
\hline $3 s 3 p\left({ }^{3} P\right) 3 d^{4} D_{7 / 2}$ & 319903.9 & 99 & & & \\
\hline $3 s 3 p\left(^{3} P\right) 3 d^{2} D_{s / 2}$ & 328958.8 & 47 & & $3 s 3 p 3 d\left(^{3} P\right.$ & $D+323 s^{3} p\left({ }^{1} P\right) 3 d^{2} D+203 p^{32} D$ \\
\hline $\left.3 s 3 p^{3} P\right) 3 d^{2} D_{3 / 2}$ & 328989.9 & 47 & & $3 s 3 p 3 d\left({ }^{3} P\right.$ & $D+323 s 3 p\left(^{1} P\right) 3 d^{2} D+203 p^{32} D$ \\
\hline $3 s^{2} 4 s^{2} s_{1 / 2}$ & 342302.8 & 99 & & & \\
\hline $\left.3 s 3 p f^{3} P\right) 3 d^{2} F_{s / 2}$ & $344307.9^{a}$ & 71 & & $3 s 3 p 3 d\left({ }^{3} P\right.$ & $F+283 s 3 p\left({ }^{1} P\right) 3 d^{2} F$ \\
\hline $\left.3 s 3 p p^{3} P\right) 3 d^{2} F_{T / 2}$ & $346073.4^{\circ}$ & 71 & & $3 s 3 p 3 d\left({ }^{3} P\right.$ & $F+283 s 3 p\left({ }^{1} P\right) 3 d^{2} F$ \\
\hline $\left.3 s 3 p x^{3} P\right) 3 d^{2} P_{3 / 2}$ & $375657.6^{\circ}$ & 78 & & $3 s 3 p 3 d t^{3} P$ & $P+163 p^{32} P$ \\
\hline $3 s 3 P\left({ }^{1} P\right) 3 d^{2} F_{7 / 2}$ & $376417.2^{a}$ & 71 & & $3 s 3 p 3 d\left({ }^{1} P\right.$ & $F+283 s 3 p\left({ }^{3} P\right) 3 d^{2} F$ \\
\hline $\left.3 s 3 p x^{1} P\right) 3 d^{2} F_{s / 2}$ & $376901.5^{\prime \prime}$ & 71 & & $3 s^{3} p 3 d\left({ }^{2} P\right.$ & $F+283 s 3 p\left({ }^{3} P\right) 3 d^{2} F$ \\
\hline $3 s 3 p\left({ }^{1} P\right) 3 d^{2} D_{3 / 2}$ & $3954920^{n}$ & 66 & & $3 s 3 p 3 d\left({ }^{1} P\right.$ & $\left.D+173 s 3 p p^{3} P\right) 3 d^{2} D+153 p^{32} D$ \\
\hline $\left.3 s 3 p x^{1} P\right) 3 d^{2} D$ & $395804.1^{\mathrm{D}}$ & 67 & & $3 s 3 p 3 d l^{1} P$ & $\left.D+173 s 3 p p^{3} P\right) 3 d^{2} D+153 p^{3}{ }^{3} D$ \\
\hline $3 s 3 p\left(^{3} P\right) 4 s^{4} P_{1 / 2}$ & 454109.8 & 99 & & & \\
\hline $3 s^{2} 4 d^{2} D_{3 / 2}$ & 454745.6 & 99 & & & \\
\hline $3 s^{2} 4 d^{2} D_{s / 2}$ & 454798.8 & 99 & & & \\
\hline $3 s 3 p\left({ }^{3} P\right) 4 s^{4} P_{3 / 2}$ & 454872.5 & 99 & & & \\
\hline $3 s 3 p\left(^{3} P\right) 4 s^{4} P_{5 / 2}^{3 / 2}$ & 456271.6 & 99 & & & \\
\hline $3 s 3 p f^{1} P y s^{2} P_{1 / 2}^{3 / 2}$ & $525043.6^{\mathrm{n}}$ & 99 & & & \\
\hline $3 s 3 p\left({ }^{1} P\right) 4 s^{2} P_{3 / 2}$ & $525191.3^{n}$ & 98 & & & \\
\hline
\end{tabular}

- Percentages lower than $5 \%$ are omitted

n New level

another new line at $1422.51 \AA$, classified as $3 s^{2} 4 d^{2} D_{3 / 2}-3 s 3 p\left({ }^{1} P\right) 4 s^{2} P_{1 / 2}$.

For the level $3 s 3 p\left({ }^{1} P\right) 4 s^{2} P_{3 / 2}$ we propose the new value $525191.3 \mathrm{~cm}^{-1}$. This level is supported by three new lines that are classified in Table I.

We report a new line at $618.72 \AA$, classified as $3 s 3 p^{22} D_{3 / 2}-3 p^{32} P_{3 / 2}$ transition. For us, the line at $804.62 \AA$, corresponds to the $3 s 3 p^{2} S_{1 / 2}-3 p^{32} P_{3 / 2}$ transition. This line has a double classification in the previous work of Ref. [22]. In the same work, some other lines also appear with double classification, but for us, the lines at $893.50 \AA, 893.63 \AA$ and $904.90 \AA$ correspond to the $3 s 3 p^{2}{ }^{2} P-3 p^{32} P$ multiplet. These transitions confirm (fairly well) the previously observed values of the $3 p^{32} P_{1 / 2}$ and ${ }^{2} P_{3 / 2}$ levels, see Ref. [22].

We report two new lines at $508.91 \AA$ and $509.12 \AA$, classified as $3 s 3 p^{22} D_{3 / 2}-3 s 3 p\left({ }^{3} P\right) 3 d^{2} D_{5 / 2}$ and $3 s 3 p^{2}{ }^{2} D_{5 / 2}-3 s 3 p\left({ }^{3} P\right) 3 d^{2} D_{3 / 2}$ transitions. These lines confirm the previously observed values of the $3 s 3 p\left({ }^{3} P\right) 3 d^{2} D$ doublet, see Ref. [22].

\section{Theoretical Interpretation}

The level structure was theoretically interpreted by a leastsquares fit of the energy parameters to the experimental level values. For this purpose the computer code developed by Cowan [29] was used.

The scaled Hartree-Fock factor was 0.85 for all parameters, except for $\zeta_{n l}$ where the scaled factor was 0.95 and for $E_{\text {ar }}$ where the scaled factor was 1.00 . These scaled factors were taken in this form because the computed energy-level intervals agree better with the experimental ones.

In order to obtain a better interpretation of the levels it was necessary to introduce the $3 s^{2} 4 p$ configuration. The results of the parametric calculations are presented in Table III.

The $\alpha$ parameter was kept free because all the levels of the $3 p^{3}$ configuration are known. The first three configuration interaction integrals were held fixed in the calculation scaled at $0.75,0.95$ and 0.95 of their Hartree-Fock values. The rest of the configuration interaction integrals were held fixed at 
Table III. Energy parameters $\left(\mathrm{cm}^{-1}\right)$ for the $3 s^{2} 3 p, 3 s^{2} 4 p, 3 p^{3}, 3 s 3 p 3 d, 3 s 3 p 4 s$ configurations of $A r V I$

\begin{tabular}{|c|c|c|c|c|}
\hline Configuration & Parametes & $\begin{array}{l}\text { Scaled } \\
\text { HI Value }\end{array}$ & Fitted Value & $\begin{array}{l}\text { Ratio Fitted Value } \\
\text { SHF Yalue }\end{array}$ \\
\hline $3 s^{2} 3 p$ & $E_{\text {av }}$ & 0 & 13738 & \\
\hline $3 s^{2} 3 p$ & $\zeta_{3 p}$ & 1307 & 1512 & 1.157 \\
\hline $3 s^{2} 4 p$ & $E_{\mathrm{av}}$ & 391438 & 393887 & 1.006 \\
\hline $3 s^{3} 4 p$ & $\zeta_{a p}$ & 394 & 394 (FIX) & \\
\hline $3 p^{3}$ & $E_{\mathrm{av}}$ & 287614 & 294987 & 1.026 \\
\hline $3 p^{3}$ & $F^{2}(3 p, 3 p)$ & 66962 & 68868 & 1.028 \\
\hline $3 p^{3}$ & $a(3 p, 3 p)$ & & -112 & \\
\hline $3 p^{3}$ & $\zeta_{3 p}$ & 1306 & 1610 & 1.233 \\
\hline $3 s 3 p 3 d$ & $E_{\mathrm{av}}$ & 323495 & 332094 & 1.027 \\
\hline $3 s 3 p 3 d$ & $F^{2}(3 p, 3 d)$ & 63506 & 63334 & 0.997 \\
\hline $3 s 3 p 3 d$ & $G^{1}(3 s, 3 p)$ & 90341 & 70384 & 0.779 \\
\hline $3 s 3 p 3 d$ & $G^{2}(3 s, 3 d)$ & 61497 & 66186 & 1.076 \\
\hline $3 s 3 p 3 d$ & $G^{1}(3 p, 3 d)$ & 78310 & 76869 & 0.982 \\
\hline $3 s 3 p 3 d$ & $G^{3}(3 p, 3 d)$ & 48916 & 44557 & 0.911 \\
\hline $3 s 3 p 3 d$ & $\zeta_{3 p}$ & 1319 & 1797 & 1.362 \\
\hline $3 s 3 p 3 d$ & $\zeta_{3}$ & 60 & 60 (FIX) & \\
\hline $3 s 3 p 4 s$ & $E_{\mathrm{av}}$ & 463784 & 475779 & 1.026 \\
\hline $3 s 3 p 4 s$ & $G^{1}(3 s, 3 p)$ & 82900 & 95501 & 1.152 \\
\hline $3 s 3 p 4 s$ & $G^{0}(3 s, 4 s)$ & 5896 & 6137 & 1.041 \\
\hline $3 s 3 p 4 s$ & $G^{1}(3 p, 4 s)$ & 7775 & 7775 (FIX) & \\
\hline $3 s 3 p 4 s$ & $\zeta_{u_{p}}$ & 1405 & 1420 & 1.011 \\
\hline \multicolumn{5}{|l|}{$\begin{array}{l}\text { Configuration } \\
\text { Interaction } \\
\text { Integrals }\end{array}$} \\
\hline $3 s^{2} 3 p-3 p^{3}$ & $R^{1}\left(3 s^{3} s, 3 p^{3} p\right)$ & 79351 & 79351 (FIX) & \\
\hline $3 s^{2} 3 p-3 s 3 p 3 d$ & $R^{2}(3 s 3 p, 3 p 3 d)$ & 92765 & 92765 (FIX) & \\
\hline $3 s^{2} 3 p-3 s 3 p 3 d$ & $R^{2}(3 s 3 p, 3 d 3 p)$ & 70775 & 70775 (FIX) & \\
\hline $3 s^{2} 3 p-3 s 3 p 4 s$ & $R^{0}(3 s 3 s, 3 s 4 s)$ & 4725 & 4725 (FIX) & \\
\hline $3 s^{2} 3 p-3 s 3 p 4 s$ & $R^{1}(3 s 3 p, 3 p 4 s)$ & 7557 & 7557 (FIX) & \\
\hline $3 s^{2} 3 p-3 s 3 p 4 s$ & $R^{0}\left(3 s^{3} p, 4 s^{3} p\right)$ & 1368 & 1368 (FIX) & \\
\hline $3 s^{2} 4 p-3 s 3 p 3 d$ & $R^{1}(3 s 4 p, 3 p 3 d)$ & 8998 & 8998 (FIX) & \\
\hline $3 s^{2} 4 p-3 s 3 p 3 d$ & $R^{2}(3 s 4 p, 3 d 3 p)$ & 10777 & 10777 (FIX) & \\
\hline $3 s^{2} 4 p-3 s 3 p 4 s$ & $R^{1}(3 s 4 p, 3 p 4 s)$ & 38098 & 38098 (FIX) & \\
\hline $3 s^{2} 4 p-3 s 3 p 4 s$ & $R^{0}\left(3 s^{4} p, 4 s^{3} p\right)$ & 6074 & 6074 (FIX) & \\
\hline $3 p^{3}-3 s 3 p 3 d$ & $R^{1}(3 p 3 p, 3 s 3 d)$ & 82900 & 82900 (FIX) & \\
\hline $3 p^{3} \quad-3 s 3 p 4 s$ & $R^{1}(3 p 3 p, 3 s 4 s)$ & 7384 & 7384 (FIX) & \\
\hline $3 s 3 p 3 d-3 s 3 p 4 s$ & $R^{2}(3 p 3 d, 3 p 4 s)$ & 2974 & 2974 (FIX) & \\
\hline $3 s 3 p 3 d-3 s 3 p 4 s$ & $R^{1}(3 p 3 d, 4 s 3 p)$ & 5010 & 5010 (FIX) & \\
\hline
\end{tabular}

- The RMS deviation of the fit is $210 \mathrm{~cm}^{-1}$ for 28 observed levels

the standard $85 \%$ of the Hartree-Fock values. The standard-deviation for the 28 observed levels was $210 \mathrm{~cm}^{-1}$.

\section{Acknowledgements}

Support from Conselho Nacional de Desenvolvimento Cientifico e Tecnológico (CNPq), Brasil, Fundação de Amparo à Pesquisa do Estado de São Paulo (FAPESP), Brasil, Comisión de Investigaciones Científicas de la Proviscia de Buenos Aires (CIC), Argentina, where J. G. Reyna Almandos and $F$. Bredice belong as researchers and $M$. Raineri as fellow, and Consejo Nacional de Investigaciones Cientificas e Tócnicas (CONICET), Argentina is gratefully acknowledged.

\section{References}

1. Moore, C. E., Atomic Energy Levels, Vol. I. Nat. Bur. Stand., Ref. Data Ser. Circ. N: 467 (U.S. GPO, Washington D.C. 1971)

2. Eriksson, K. B. S. and Isberg, H. B. S., Ark. Fys. 23, 527 (1963)

3. Shenstone, A. G. Proc. Roy. Soc. A261, 153 (1961).

4. Magnusson, C. E. and Zetterberg, P. O., Phys. Scr. 15, 237 (1977).
5. Martin, W. C., Zalubas, R. and Musgrove, A. J., J. Phys. Chem. Rer. Data 19, 821 (1990).

6. Phillips, L. W. and Parker, W. L., Phys. Rev. 60, 301 (1941).

7. Fawceth, B. C. Jones, B. B. and Wilson, R., Proc. Phys. Soc. 78, 1223 (1961).

8. Schönheit, E, Optik 23, 409 (1966)

9. Ekberg, J. O. and Svensson, L A, Phys. Scr. 2,283 (1970).

10. Fawceth, B. C., Ridgeley, A. and Bromage, G. E., Phys. Scr. 18, 315 (1978).

11. Livingston, A. E, Pinnington, E. H, Irwin, J. G, Kernahan, J. A. and Brooks, R. L., J. Opt. Soc. Am. 71, 442 (1981).

12 Buchet-Poulizac, M. C. Buchet, J. P. and Ceyzeriat, P., Nucl. Instr. and Meth. 202, 13 (1982).

13. De-Ye, J., Li-Kang, S, Li-Zeng, Z and Wen-Shu, W, Chin. J. Phys. 33, 508 (1984).

14. Fawcett, B. Cn At. Data and Nucl. Data Tables 28, 557 (1983).

15. Huang, K.-N., At. Data and Nucl. Data Tables 34, 1 (1986).

16. Sugar, J. and Kaufman, V, J. Opt. Soc. Am. B3, 704 (1986)

17. Sugar, J. and Kaufman, V., Phys. Scr. 34,797 (1986).

18. Träbert, E, Hutton, R. Engström, L., Bliman, S. L., Berry, H. G. and Kurtz, C, Phys. Lett. A129, 381 (1988).

19. Träbert, E, Heckmann, P. M., Hutton, R. and Martinson, I., J. Opt. Soc. Am. BS, 2173 (1988).

20. Lesteven-Vaïsse, I, Folkmann, F, Ben Sitel, A, Chantepie, M. and Lecler, D., Phys. Scr. 38, 45 (1988).

Physica Scripta 45 
21. Engström, L., Reistad, N., Jupén, C. and Westerlind, M., Phys. Scr. 39, 25. Jupén, C., Denne, B. and Martinson, I., Phys. Scr. 41, 669 (1990). 66 (1989).

22. Pinnington, E. H, Ge, Z.-Q, Ansbacher, W., Kernahan, J. A. and Goselin, R. N, Phys. Scr. 39, 321 (1989).

23. Redfors, A. and Litzen, U., J. Opt. Soc. Am. B6, 1447 (1989).

26. Gallardo, M., Bredice, F, Raineri, M. and Reyna Almandos, J. G. Appl. Opt. 28, 4513 (1989).

27. Kelly, R. L, J. Phys. Chem. Ref. Data 16, Suppl. 1 (1987)

24. Thombury, J. F., Hibbert, A. and Träbert, E., Phys. Scr. 40, 472 (1989).

28. Pettersson, S.-G., Phys. Scr. 26, 296 (1982)

29. Cowan, R. D. "The Theory of Atomic Structure and Spectra" (University of California Press, Berkeley, U.S.A. 1981). 